\title{
ДИАГНОСТИКА И ОПЕРАТИВНОЕ ЛЕЧЕНИЕ ДИВЕРТИКУЛОВ
}

\author{
А. С. Гырхларова, С. А. Гаджиев \\ Азербайджанский медицинский университет, г. Баку, Республика Азербайджан \\ DIAGNOSIS AND OPERATIVE TREATMENT OF DIVERTICULA
}

\author{
A. S. Gyrhlarova, S. A. Gadzhiyev \\ Azerbaijan Medical University, Baku, Republic Azerbaijan
}

\section{Ресрерат}

Обобщены результаты лечения 20 пациентов по поводу изолированных и сочетанных дивертикулов желудка, двенадцатиперстной кишки (ДПК), тонкой и толстой кишки.

Предложен метод диагностики дивертикулов по данным рентгенологического исследования с применением зонда Рильсина. Разработана и апробирована методика устранения дивертикулов путем наложения гофрирующих швов, позволившая избежать рецидивирования дивертикулов.

Ключевые слова: дивертикулы органов пищеварительной системы; диагностика; оперативное лечение.

\section{Abstract}

Results of treatment of 20 patients, suffering isolated and concomitant diverticula of stomach, duodenum, small and large intestine, were summarized.

Diagnostic method for diverticula, basing on data of roentgenological investigation, using Rilsin`s probe, was proposed.

Procedure for the diverticula elimination, using corrugating suturing, permitting to escape the diverticula recurrence, was elaborated and tested.

Keywords: diverticula of the gut; diagnosis; operative treatment.

Дивертикулы, возникающие в полых органах пищеварительной системы, различают по локализации, выраженности, частоте выявления, тяжести осложнений, сочетанию с другими заболеваниями [1 - 3]. Дифференциальная диагностика дивертикулов, целенаправленно проведенные клинические исследования и их интерпретация не систематизированы [2, 4-7]. Правильно выбранная лечебная тактика, выбор сроков ее применения, метода оперативного вмешательства, анализ ближайших и отдаленных результатов лечения недостаточно отражены в литературе $[1,4,5,8,9]$.

При сочетании дивертикулов с другими заболеваниями органов пищеварительной системы многие моменты не выяснены. Разнообразные проявления дивертикулярной болезни и ее сочетание с другими заболеваниями органов пищеварительной системы, в том числе желудка, ДПК, тонкой и толстой кишки, усложняют клиническую диагностику $[6,10]$. При сочетанной патологии выполнение операции не обеспечивает устранение основного заболевания, что обусловливает повторное обращение больных за медицинской помощью, при обнаружении в последующем дивертикула требуется выполнение повторной операции $[5,11]$.

Оптимизация и разработка усовершенствованных методов диагностики дивертикулов на различных участках пищеварительной системы, выявление их строения, истинных размеров, уточнение формы и размеров входной части отдельных дивертикулов и объединение их в одну систему являются актуальной задачей [9]. Частота выявления дивертикулов в сочетании с другими заболеваниями органов пищеварительной системы, поиск причин их образования, а также разработка тактики лечения актуальны [1].

Важной является разработка метода устранения дивертикулов со вскрытием просвета органа, в котором он возник, и неполного его удаления. Объективная оценка ближайших и отдаленных результатов разработанной и внедренной в практику операции поможет решить ряд вопросов $[8,10]$.

Цель исследования: улучшение результатов лечения дивертикулов с применением оптимальной диагностики и хирургического лечения.

\section{МАТЕРИАЛЫ И МЕТОДЫ ИССЛЕДОВАНИЯ}

Работа проведена в хирургическом отделении Городской клинической больницы № 4 им. Ф.А. Эфендиева, учебно-хирургической клинике Азербайджанского медицинского университета, Городской центральной клинической больнице № 1 и рентгенологическом отделении клиники Mediko-Hospital. B 2005 - 2016 гг. обследовали 20 больных с сочетанными дивертикулами желудка, ДПК и толстой кишки.

У всех больных детально анализировали данные анамнеза, жалобы, результаты объективного обследования. Рентгенологическое исследование проводили с видеозаписью, контрастной эзофагогастродуоденографией и ирригографией. Для обнаружения дивертикулов желудка и ДПК исследование проводили по оригинальной методике. Исследование толстой кишки проводили не только для обнаружения дивертикулов, но и оценки состояния кишечника до появления дивертику- 
лярной болезни, до и после применения спазмолитиков. Первичными признаками наличия дивертикулов являлись: раздражение толстой кишки, пневматоз толстой кишки, патологическая сегментация, пилообразный рисунок, асимметрия гаустр ободочной кишки, уменьшение диаметра толстой кишки, спазм сфинктеров, тонус кишечника, внутрикишечное давление, ригидность и многочисленность складок. Больным также проводили гастродуоденофиброскопию, колоноскопию, брали биоптаты для гистологического исследования. Рентгенологические и эндоскопические исследования проводили в ближайшем и отдаленном периоде.

Метод диагностики дивертикулов. Стандартная контрастная рентгенография и эндоскопическое исследование, применяемые при выявлении заболеваний пищеварительной системы, даже проведение контрастной рентгенографии с видеозаписью, не всегда позволяют установить правильный диагноз.

Мы впервые при диагностике дивертикулов применили зонд Рильсина, используемый при энтерографии. Во время рентгенологического исследования для четкости изображения и легкости проведения контрастной рентгенографии на фоне искусственной гипотонии больной проглатывает зонд. Конец зонда подводят к воротам дивертикула и, регулируя зонд извне, через входную часть дивертикула вводят его в просвет. Через зонд в просвет дивертикула вначале вводят контрастное вещество, потом вдувают воз-

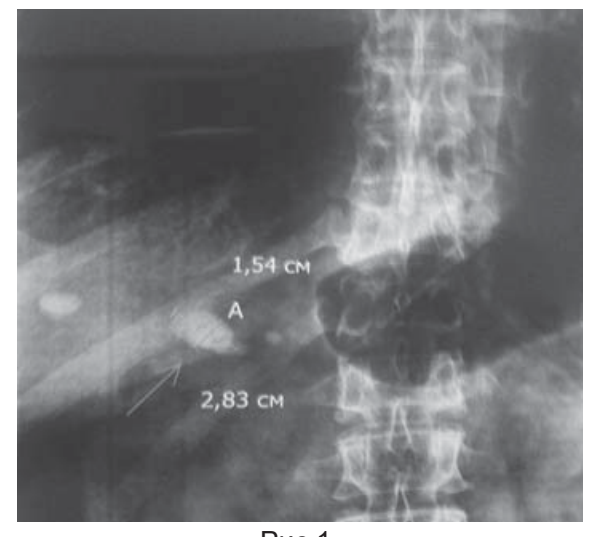

Рис 1.

Обычная гастрографияя дух, что обеспечивает полное открытие шейки и полости дивертикула. В этот момент уточняют форму, размеры, подвижность стенки, расположение и размеры ворот дивертикула. Приводим клиническое наблюдение.

Больной Г., 58 лет. В течение 20 лет проводили консервативное лечение по поводу гастрита, холецистита, панкреатита. Проведена контрастная видеорентгенография пищевода, желудка и ДПК. Наряду с конкрементами в желчном пузыре, выявлено скопление контрастного вещества в правой подреберной области, также выявлены скользящая грыжа пищеводного отверстия диафрагмы, рефлюкс-эзофагит, хроническая непроходимость ДПК. Определить связь между желудком или ДПК и скоплением контрастного вещества не было возможным. Эта связь также не была установлена по данным видеогастродуоденофиброскопии. В связи с этим с применением зонда Рильсина и соблюдением указанных правил больному проведена контрастная рентгенография с видеозаписью.

Установлено, что дивертикул образовался из задней стенки препилорической части желудка. Получена полная информация о дивертикуле (рис. 1, 2).

Во время видеоирригографии в свободном крае поперечной ободочной кишки выявлены два дивертикула. В последующем у всех больных при предположении о наличии дивертикула использовали зонд Рильсина, чем достигнут успешный результат.

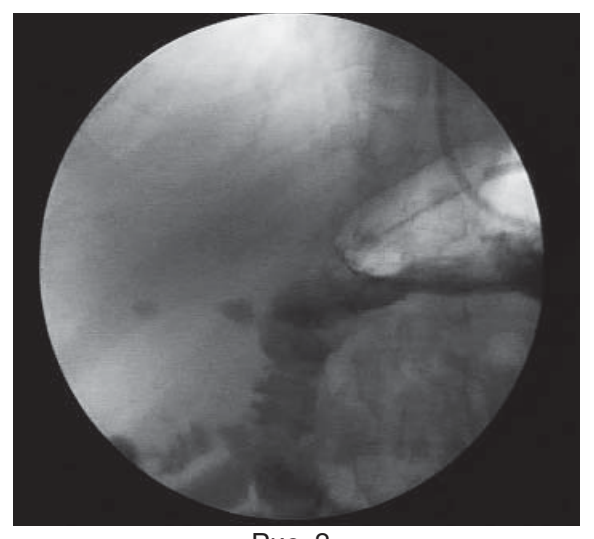

Рис. 2.

Гастрография с зондом Рильсина.
Методика устранения диверти кула. Трудное с технической точки зрения расположение органов, где возник дивертикул, требует выполнения широких операций, а при наложении швов возможны деформация органов, а также скопление массы, суживающей просвет этих органов. Вследствие этого приходится выполнять широкую резекцию части органа, в которой образовался дивертикул. Сочетанные дивертикулы желудка, ДПК и толстой кишки требуют проведения широких операций на нескольких органах, во избежание таких вмешательств необходимо использование более простых и надежных методов устранения дивертикула. Предложенная нами методика устранения дивертикула с наложением гофрирующих швов соответствует всем указанным требованиям. Значение и отличие этой методики от других способов устранения дивертикула с применением гофрирующих швов (по А. Г. Земляному) заключается в следующем: во время оперативного вмешательства в мышечной оболочке, где расположены ворота дивертикула, обнаруживают фиброзное кольцо. В желудке посредством продольного разреза достигают верхнего и нижнего полюсов дивертикула, проходят через серозную и мышечную оболочку, на оба полюса дивертикула накладывают шов, фиксируют его ворота, нити растягивают в стороны. При этом дно и тело дивертикула имеют вид волнообразных слоев. Последующие швы накладывают в продольном направлении и, проходя через волнообразные складки, охватывают оба края фиброзного кольца. До полного закрытия дивертикула швы повторяют на расстоянии 0,5 см. В конечном счете, дивертикул полностью прошивают гофрирующими швами. Соединение фиброзных краев мышц надежно закрывает ворота дивертикула, при наложении швов по волнообразным складкам стенки путем гофрирования в слизистой оболочке органа создается складчатая поверхность, а не грубый конгломерант, выпячивающийся в просвет органа. Применение предложенного метода позволяет восстановить целостность всех 
слоев стенки органа, а гофрирующие швы предупреждают рецидивирование дивертикулов. При операциях по устранению дивертикулов ДПК, тонкой и толстой кишки швы накладывают не в продольном, а в поперечном направлении.

После наложения гофрирующих швов резецированной части толстого кишечника его просвет вскрываЮт, при осмотре швов со стороны слизистой оболочки видно, что участок с входной частью дивертикула полностью восстановлен и не отличается от интактных участков.

\section{РЕЗУЛЬТАТЫ \\ И ИХ ОБСУЖДЕНИЕ}

Соотношение частоты выявления сочетанных дивертикулов представлено в табл. 1.

Несмотря на различные размеры дивертикулов желудка, ДПК и толстой кишки, мы сгруппировали их по количеству (табл. 2).

По результатам исследования, установлено более частое возникновение дивертикулов в желудке и медиальной части ДПК (у 50\% больных).

При обследовании у пациентов, помимо дивертикулов, выявлены сопутствующие заболевания: рефлюкс-эзофагит со скользящей грыжей пищеводного отверстия диафрагмы, рефлюкс-гастрит, хроническая непроходимость ДПК, висцероптоз с синдромом хронического запора, желчнокаменная болезнь, хронический панкреатит. Повышение давления в желудке и ДПК способствует формированию грыжи пищеводного отверстия диафрагмы, гастрита и рефлюкс-эзофагита. Учитывая зависимость двигательной

\section{REFERENCES}

1. Alekseenko AA. K khirurgicheskomu lecheniyu divertikulov zheludka i dvenadtsatiperstnoy kishki. Khirurgiya. 1964;(9):48-53. [In Russian].

2. Andrianovskiy AF. K voprosu o divertikuleze dvenadtsatiperstnoy kishki. Trudy Kazanskogo instituta usovershenstvovaniya vrachey. Kazan, 1961;(15):173-83. [In Russian].

3. Arungazyev VYu. Divertikuly dvenadtsatiperstnoy kishki. In: Trudy nauchno-issledovatelskogo instituta. Sverdlovsk, 1993.135-55. [In Russian].

4. Abrikosov Al, editor. Rukovodstvo po patologicheskoy anatomii. Moskva: Medgiz, 1957;4, 138 s. [In Russian].

5. Agzamkhodzhaev SM, Yarugskiy EE. Lechenie duodenalnykh divertikulov. Medicinskiy zhurnal Uzbekistana. 1989;(10):41-3. [In Russian].

Таблица 1. Частота обнаружения дивертикулов желудка и дПк с дивертикулами толстой кишки

\begin{tabular}{|c|c|c|c|c|}
\hline \multirow{2}{*}{ Желудок } & \multirow{2}{*}{ Количество } & \multicolumn{3}{|c|}{ Ободочная кишка } \\
\hline & & восходящая & поперечная & нисходящая \\
\hline Проксимальная часть & 2 & - & 1 & 1 \\
\hline Тело & 2 & - & 2 & - \\
\hline Препилорическая часть & 4 & 2 & 2 & - \\
\hline \multicolumn{5}{|l|}{ дПк } \\
\hline $\begin{array}{l}\text { Верхняя часть } \\
\text { (задняя стенка) }\end{array}$ & 2 & - & 2 & - \\
\hline $\begin{array}{l}\text { Нисходящщя часть } \\
\text { (медиальная) }\end{array}$ & 4 & 2 & - & 2 \\
\hline Горизонтальная часть & 6 & 2 & 2 & 2 \\
\hline
\end{tabular}

Таблица 2. Количество и размеры дивертикулов желудка, дпк и толстой кишки

\begin{tabular}{|cccccc|}
\hline \multicolumn{2}{|c}{ Дивертикулы желудка } & \multicolumn{2}{c|}{ Дивертикулы ДПК } & \multicolumn{2}{c|}{ Дивертикулы толстой кишки } \\
\hline Количество & Размеры, см & Количество & Размеры, см & Количество & Размеры, см \\
\hline 4 & $2,0 \times 1,5$ & 3 & $1,0 \times 0,8$ & 6 & $2,0 \times 1,5$ \\
\hline 2 & $3,5 \times 2,0$ & 3 & $1,5 \times 1,0$ & 6 & $3,0 \times 2,0$ \\
\hline 2 & $4,0 \times 3,5$ & 6 & $2,0 \times 1,5$ & 8 & $3,5 \times 2,5$ \\
\hline
\end{tabular}

активности ДПК, тонкой и толстой кишки от центра, расположенного в нисходящей части ДПК, становится понятным наличие застойных явв кишечнике при дивертикуости является причиной атрофии входной части дивертикула.

После лечения через 3, 6, 12, 24 и 36 мес повторно проводили контрастную рентгенографию и эндоскопию с видеозаписью. У всех паов слизистая оболочка на месте оперативного вмешательства без патологических изменений.

\section{выводы} дивертикулов, образующихся на различных участках пищеварительной системы, является кон- трастная телевидеография с применением зонда Рильсона в условиях гипотонии. Исследование дивертикулов толстой кишки следует проводить после оценки признаков преддивертикулярного состояния.

2. При планировании операции по поводу устранения дивертикулов необходимо учитывать наличие сочетанных заболеваний органов пищеварительной системы с последующим планированием их коррекции.

3. По поводу единичных или сочетанных дивертикулов, щадящей операцией можно считать предложенный нами способ с сохранением целостности органа без вскрытия его просвета, где возник дивертикул. Хорошие результаты предложенного способа операции подтверждают целесообразность его широкого применения.
6. Alekseenko AA. Divertikuly dvenadtsatiperstnoy kishki. Klinika, diagnostika i lechenie. Klinicheskaya meditsina. 1964;(2):26-32. [In Russian].

7. Andreytseva OI. Diagnostika i lechenie okolososochkovykh divertikulov dvenadtsatiperstnoy kishki [thesis]. Rostov na Donu; 1993. 23 s. [In Russian].

8. Andrianovskiy AF. Divertikuly dvenadtsatiperstnoy kishki. Kazanskiy medicinskiy zhurnal. 1961:36-9. [In Russian].

9. Babiets AP. Klinika, diagnostika i lechenie divertikulov dvenadtsatiperstnoy kishki. Klinicheskaya khirurgiya. 1969;81-2. [In Russian].

10. Babiets AP. Klinika, diagnostika i pokazaniya k khirurgicheskomu lecheniyu divertikulov dvenadtsatiperstnoy kishki [thesis]. Vinnitsa; 1969. $22 \mathrm{~s}$. [In Russian].

11. Alekseenko AA. Klinika, diagnostika i khirurgicheskoe lechenie divertikulov zheludka i dvenadtsatiperstnoy kishki [thesis]. Donetsk 1965, 23 s. [In Russian]. 